How to reference this article

Ondelli, S. \& Romanini, F. (2018). Norma interiorizzata e uso: un'indagine preliminare su parlanti italiani. Italica Wratislaviensia, 9(1), 185-207.

DOI: http://dx.doi.org/10.15804/IW.2018.09.10

\author{
Stefano Ondelli \& Fabio Romanini \\ Università di Trieste
}

\title{
NORMA INTERIORIZZATA E USO: \\ UN'INDAGINE PRELIMINARE SU PARLANTI ITALIANI
}

\section{INTERNALISED NORMAND USAGE: AN INTRODUCTORY SURVEY ON ITALIAN NATIVE SPEAKERS}

\begin{abstract}
Over 30 years after the publication of the list of 35 traits characterising the "medium [i.e., between formal and informal] register of Italian", this sociolinguistic label is commonly accepted, although many researchers have improved and modified its description. However, teachers in Italian schools have not proven to be particularly sensitive to the widening gap between norm and usage and still teach a variety of Italian that is not found anywhere else but in the classroom. Unfortunately, insufficient research has been conducted so far on the differences between the norms that Italian native speakers learn in their school years and their actual linguistic behaviour. Which traits of the IUM (italiano dell'uso medio) pass unnoticed, which are detected but considered acceptable, and which are classified as mistakes are still up for debate.

This study illustrates a survey involving native speakers of Italian coming from different geographical areas and social backgrounds who were invited to comment on the language used in newspaper articles containing IUM traits. Of course, this survey is part of a larger research project and is not considered to be exhaustive; however, it proves useful in testing the most effective procedures to administer the questionnaires and select the informants. In addition, preliminary results show a strong influence of the linguistic norm taught at school on the assessment not only of IUM traits but also of a range of other traits contributing to the linguistic reference model used by Italian native speakers.
\end{abstract}

Keywords: linguistic norm, language usage, neo-standard Italian, registers of Italian, sociolinguistics 


\section{PREMESSE TEORICHE ${ }^{1}$}

Sono passati 30 anni dalla pubblicazione della Sociolinguistica dell' $i-$ Staliano contemporaneo di Gaetano Berruto (1987), il volume che si è imposto prima come livre de chevet e poi come lettura imprescindibile per chi si occupa delle varietà dell'italiano in ottica sincronica. In particolare, tramite l'etichetta di neostandard, lo schema sociolinguistico proposto da Berruto ribadiva l'esistenza di un registro medio della lingua distinto dalla supernorma stabilita dalla tradizione e largamente condiviso da ampi strati di popolazione. Questo registro, due anni prima, era stato descritto nei suoi 35 principali tratti da Francesco Sabatini (1985), che a sua volta aveva proposto la denominazione di Italiano dell'uso medio (di seguito IUM). A prescindere dalle etichette, precedenti o successive, come quella di italiano tendenziale di Mioni (1983) o di italiano senza aggettivi di Castellani (1991), talvolta non perfettamente sovrapponibili nella delimitazione del fenomeno, numerosi sono gli studiosi che si sono occupati di precisarne le caratteristiche: possiamo citare Mirko Tavoni (2002) e Raffaele Simone (1993) per quanto concerne la ristrutturazione del sistema verbale (ma anche D'Achille 2010); Michele Cortelazzo (2000a, pp. 16-21) sui pronomi; Lorenzo Renzi (2012, 37-76) soprattutto sulle dislocazioni; Paolo D'Achille (2014 e D'Achille, Proietti \& Viviani 2005) sulla frase scissa.

Tutti questi contributi erano tesi a definire e delimitare quello che può essere considerato il risultato finale della forte accelerazione che ha interessato il riassestamento della norma linguistica in Italia a partire dal secondo dopoguerra. I cambiamenti sociali dovuti all'industrializzazione e alle migrazioni interne, unitamente all'alfabetizzazione (con tutti i limiti che persistono tutt'oggi) e all'italianizzazione della popolazione, così ben descritti da Tullio De Mauro (2014), hanno determinato la grande pressione del parlato sulla scrittura, fino a quel momento unica

1 Questo contributo è il frutto della collaborazione dei due autori, che ringraziano Valeria Mantese e Lorenzo Penta per l'aiuto nella predisposizione del sondaggio e nella raccolta dei dati; tuttavia specifichiamo che Stefano Ondelli ha redatto i paragrafi 1,2 e 4, e Fabio Romanini il paragrafo 3. 
varietà depositaria della norma. A voler semplificare al massimo, l'IUM si configura infatti come il risultato dell'accoglienza o della riammissione nei testi scritti di tratti linguistici tipici dell'italiano parlato (sulla persistenza del parlato nella scrittura nella storia della lingua italiana cfr. D'Achille 2006). Questa permeabilità si acutizza ulteriormente all'inizio del nuovo millennio, quando Antonelli (2011) registra una nuova fase di oralizzazione e abbassamento dei registri in tutti i tipi di testo proprio nel momento in cui, grazie all'avvento dei nuovi media, la scrittura torna a svolgere un ruolo centrale per la lingua nazionale anche nella comunicazione quotidiana.

Tuttavia, il problema del modello di riferimento non sembra essere affatto risolto. Se da una parte si può dire che la letteratura abbia cessato di fornire il canone linguistico e che la "buona scrittura" sia piuttosto da ricercarsi nei testi giornalistici di qualità (come già affermato da Dardano, 1994, p. 34; e poi ribadito da Antonelli, 2011, p. 51) in quanto depositari della nuova medietà linguistica, la scuola quasi non se ne accorge. Al di là delle prescrizioni contenute nelle grammatiche scolastiche, la norma interiorizzata formatasi negli anni della scuola (Serianni, 2006, p. 43) e la norma sommersa individuata dalle correzioni dei testi degli alunni da parte dei docenti (Serianni, 2007) contribuiscono al persistere dello "scolastichese" (i cui tratti sono stati descritti da Cortelazzo, 2000 b, 2012) non solo in classe, ma anche ogniqualvolta il parlante si trovi a gestire o valutare un testo, soprattutto se scritto (cfr. anche Antonelli, 2007, p. 48 e Serianni \& Benedetti, 2009).

La necessità di tenere conto della grande ricchezza e variabilità degli usi della lingua contemporanea e nel contempo di fare affidamento su regole di riferimento stabili non si limita all'ambito scolastico: per i linguisti le ripercussioni possono essere importanti nella definizione stessa di parlante nativo dell'italiano (cfr. Acquaviva, 2000; Berruto, 2012) e nella scelta di un termine di paragone in qualsiasi tipo di analisi linguistica, soprattutto se di tipo quantitativo (p. es. Ondelli \& Pontrandolfo, 2014). In effetti, se l'IUM può essere oggi considerato lo standard nel senso della frequenza statistica, il giudizio si fa più difficile quando si passa a considerare $\mathrm{i}$ concetti di correttezza grammaticale e neutralità di registro. 
Il problema sembra almeno in parte risiedere anche nella diversa natura dei 35 tratti a suo tempo individuati da Sabatini (anche se poi ridotti a 14 nel 1990) e nella conseguente diversa accettabilità da parte dei parlanti. Senza voler abusare dell'esercizio della microautobiografia linguistica (Renzi, 2012, cap. 2), due parlanti che, come gli autori di questo articolo, stavano affrontando il percorso di formazione scolastica negli anni in cui Sabatini e Berruto pubblicavano i loro studi, esprimono giudizi assai diversi sui vari tratti. A prescindere dagli aspetti fonetici, che ovviamente sfuggono al parlante non esperto, o dai fenomeni riconducibili alla variazione in diatopia, come l'uso di codesto, costì e costà, alcuni tratti potevano essere percepiti come desueti già 30 anni fa (in Isvizzera e in Ispagna), fino a rasentare l'agrammaticalità (le camicie che ho stirate), mentre altri sfuggono alla percezione (la minor frequenza della $d$ eufonica, ma anche le concordanze ad sensum, cfr. Ondelli \& Viale 2010) o sono portatori di marche diamesiche o diafasiche (gli per loro). Talvolta le difficoltà nel giudizio discendono dall'effettiva complicatezza delle regole dell'italiano (come nel caso dell'alternanza tra congiuntivo e indicativo, ma anche tra perfetto semplice e composto), mentre accanto a sanzioni certe (Marco è un ragazzo che ci si può fidare), emergono anche costrutti che paiono praticamente insostituibili persino nello scritto, come certi verbi procomplementari (per fare un tavolo ci vuole un fiore).

Anche Massimo Palermo, in uno studio sull'accettabilità dei tratti dell'IUM, decide di non considerare

fenomeni estremi, sia nel caso di piena accettazione del tratto "innovativo" (per es. il passaggio a un sistema di dimostrativi bipartito, con esclusione di codesto, il cui uso è limitato a usi regionali o burocratici), sia, sul versante opposto, nel caso di fenomeni ancora decisamente marcati come substandard ( $m e$ in funzione di soggetto, periodo ipotetico costruito col doppio condizionale, uso del clitico $c i$ con valore dativale) (Palermo, 2010, p. 243).

Il tentativo di Palermo è assai interessante ma circoscritto programmaticamente a valutare l'atteggiamento di 79 insegnanti della scuola primaria e secondaria della provincia di Siena (con 56 toscani, emerge una 
certa concentrazione in diatopia) e 92 partecipanti a corsi di formazione post lauream per l'insegnamento dell'italiano L2. È dunque probabile che soprattutto il secondo gruppo, come il piccolo gruppo di controllo di 8 studenti di italiano in un'università tedesca, sia ben consapevole della questione della ristandardizzazione linguistica. L'altro gruppo di controllo (29 adulti laureati operanti a Roma in settori diversi dall'insegnamento) non è diastraticamente rappresentativo della popolazione italiana. Il materiale linguistico è stato presentato sotto forma di una o due brevi frasi contenenti il tratto sottoposto a indagine e il giudizio doveva essere espresso scegliendo una tra cinque possibilità in un gradatum che andava dalla totale inaccettabilità alla piena accettabilità, con tre gradi intermedi (accettabile nel parlato formale, nel parlato informale e nel parlato o nello scritto di media formalità) che richiedevano una notevole competenza linguistica e comportavano (a nostro parere) un margine di incertezza nella definizione di "media formalità".

\section{MATERIALI E METODI}

L'obiettivo del nostro studio è sondare il grado di accettabilità di alcuni tratti dell'IUM presso un campione il più possibile variato di popolazione, evitando di sollecitare la censura linguistica che potrebbe essere stimolata se si attira l'attenzione degli informanti sul tratto in esame. Abbiamo dunque deciso di sottoporre a 15 gruppi di 10 intervistati ognuno brevi estratti di articoli pubblicati tra il 2015 e il 2016 da Corriere della Sera e Repubblica e di chiedere un commento libero sulla loro qualità linguistica. Poiché valutare tutti i testi e tutti i tratti del campione avrebbe comportato un impegno eccessivamente gravoso in termini di tempo e attenzione, ciascun gruppo ha valutato due testi diversi (per un totale di 30), contenenti da un minimo di uno solo a un massimo di cinque tratti. Ogni intervistato ha fornito alcune informazioni su di sé (sesso, provenienza, età, titolo di studio e professione), ha dedicato qualche minuto a leggere i due brani, della lunghezza media di una dozzina di righe, e ha espresso commenti liberi sulla lingua utilizzata senza essere guidato da indicazioni precise. È quindi capitato che i tratti dell'IUM siano stati segnalati oppure siano passati inosservati, mentre talvolta si 
sono raccolti commenti anche su aspetti che non formavano l'oggetto della nostra indagine (cfr. § 4).

La scelta è caduta sugli articoli di giornale per le considerazioni svolte sopra sulla medietà linguistica, pur nella consapevolezza delle caratteristiche proprie della scrittura giornalistica. Peraltro, la presenza del virgolettato, potenzialmente mimetico dell'oralità, all'interno di vari brani e l'appartenenza a tipologie testuali diverse (p. es. interviste o anche cronache sportive), talora suscettibili di ammettere uno stile marcatamente colloquiale, agivano innegabilmente come fattori di disturbo.

Molti testi sono stati selezionati proprio perché presentavano al loro interno i tratti sottoposti a indagine; in altri casi è stato invece necessario introdurli ad hoc. Già la maggiore o minore frequenza, o la completa assenza, di certi costrutti dal materiale estratto dagli archivi elettronici delle due testate è indicativa della loro maggiore o minore accettabilità nell'italiano dei giornali: se non c'è stata alcuna difficoltà a reperire articoli caratterizzati dall'oscillazione tra indicativo e congiuntivo o dalle frasi scisse, l'inserimento a posteriori è risultato imprescindibile per gli al posto di le, per il che polivalente introduttore di una frase relativa nei casi obliqui e per la concordanza ad sensum. Inoltre, anche l'accordo del participio passato con l'oggetto in posizione antecedente sotto forma di pronome relativo (che non è considerato tratto caratterizzante dell'IUM, ma che abbiamo inserito come controprova) è risultato del tutto assente dagli articoli di giornale.

Tra tutti quelli individuati da Sabatini (1985) e Berruto (1987), $\mathrm{i}$ tratti indagati hanno riguardato:

- per la morfologia pronominale: gli per le e loro; tutti i casi di che polivalente; il ci attualizzante; questo/quello per cio; il rafforzamento dei dimostrativi del tipo questo qui e quello li e gli impieghi del dimostrativo desemantizzato, del tipo occorre affrontare tutti quei problemi che oggi assillano i giovani;

- per la morfologia verbale: indicativo pro congiuntivo; concordanze ad sensum e accordo del participio passato col pronome relativo;

- per l'ordine dei costituenti della frase: dislocazione a sinistra e destra; frase scissa e pseudoscissa; $c$ 'è presentativo. 
Una volta scelti i testi, è stato aperto un sondaggio online tramite il servizio Surveymonkey (it.surveymonkey.com) che, tra le altre cose, ha permesso di controllare il tempo di risposta, utile a scartare gli informanti che hanno risposto in meno di 10 minuti e che quindi, presumibilmente, non hanno dedicato un tempo sufficiente alla valutazione (tempo medio di risposta: 30 minuti).

I 10 componenti dei 15 gruppi sono stati selezionati in modo tale da garantire la massima eterogeneità. Anche se il sondaggio online già di per sé presuppone che i 150 soggetti intervistati abbiano una certa dimestichezza con i media elettronici, abbiamo cercato di garantire la variazione in diatopia (81 persone dal Nord Italia, 15 dalla Toscana e 54 da Sud e isole) e nel genere (84 donne e 66 uomini). I criteri di selezione sono stati principalmente due: l'età e l'occupazione. Partendo dal presupposto che il conservatorismo linguistico possa crescere con l'età, abbiamo incluso 75 soggetti di età compresa tra i 18 e i 25 anni e altri 75 tra i 50 e i 65 anni. Per evitare di registrare competenze specifiche nelle questioni linguistiche, nessuno degli intervistati era studente di lingua o letteratura (italiana o straniera) o insegnante di italiano o di una lingua straniera in qualsiasi tipo di scuola, anche se la fascia d'età 50-65 anni comprendeva alcuni docenti di altre materie, in particolare professori di economia aziendale e matematica. I grafici che seguono si riferiscono ai dati assoluti e riassumono la composizione del campione sottoposto a sondaggio.

Grafico 1: Distribuzione in diatopia

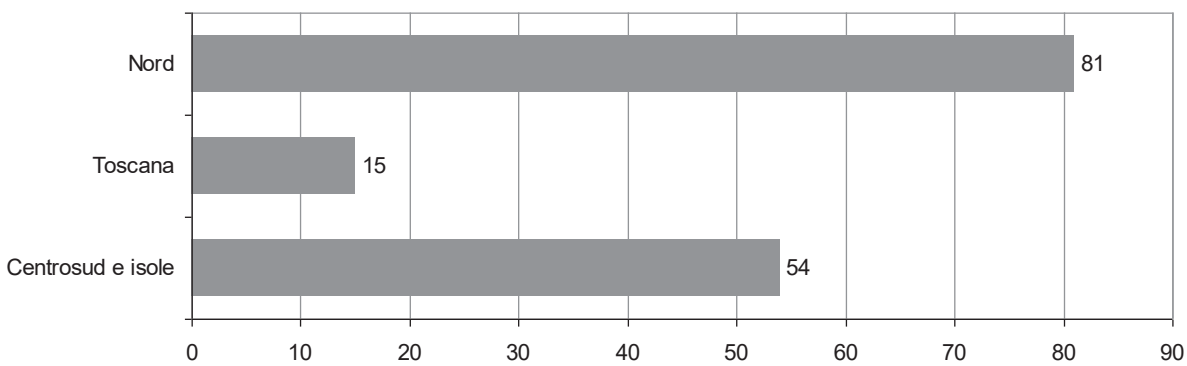


Grafico 2: Distribuzione per scolarità

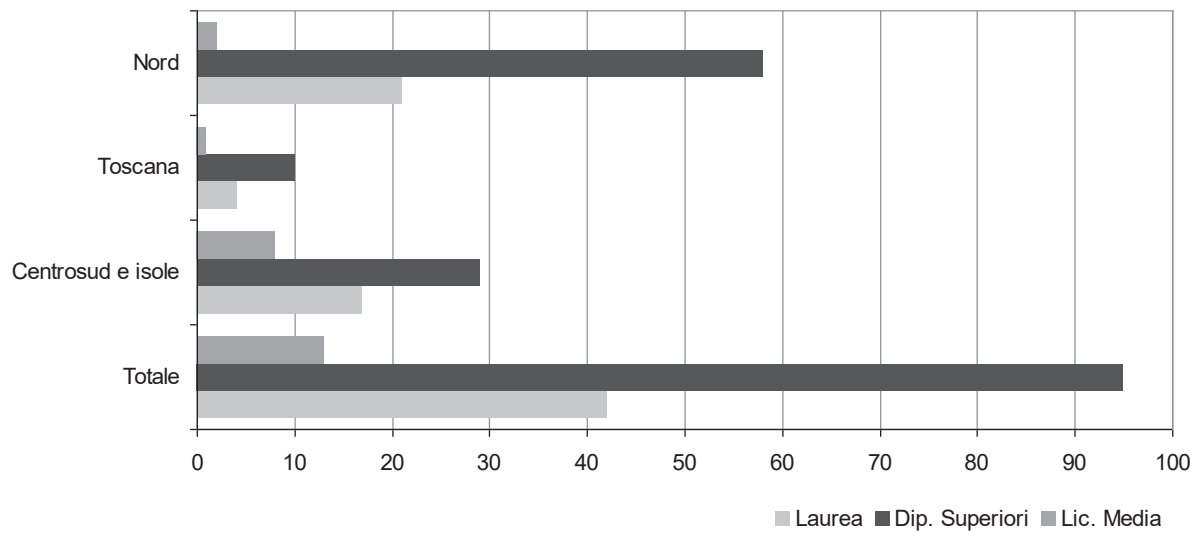

Grafico 3: Distribuzione per età

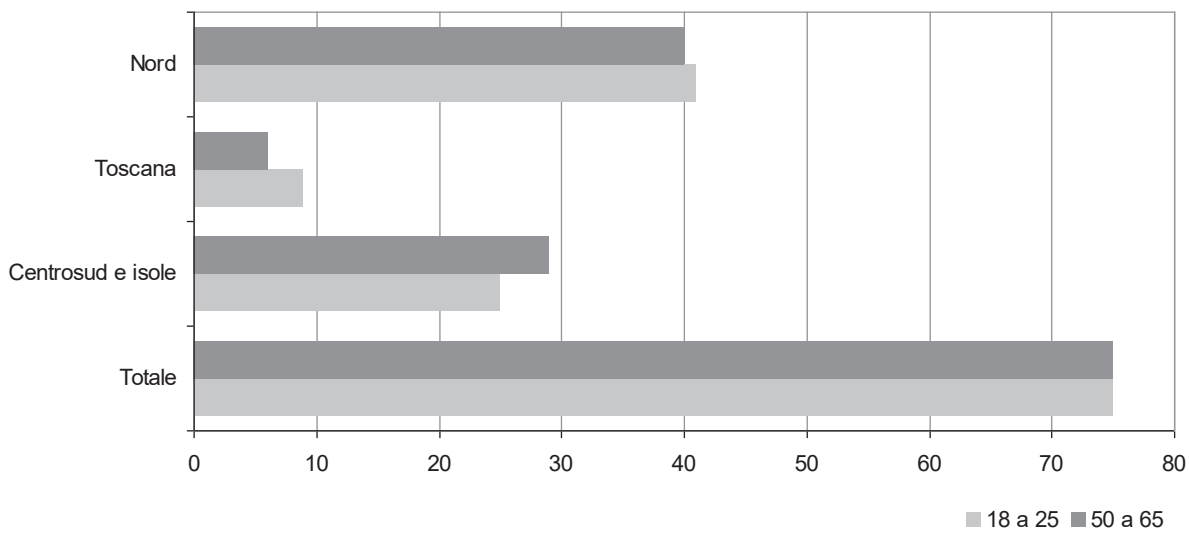

\section{IL GIUDIZIO DEI PARLANTI}

Per motivi di spazio non è possibile qui dare conto di tutti i tratti indagati, perciò saranno presentati i casi che hanno ricevuto un giudizio di accettabilità più elevato. Nei paragrafi che seguono, ciascun esempio riportato è seguito dal numero di censure fornite dai dieci informanti che giudicavano il testo. Le censure riportate si riferiscono esclusivamente al tratto in esame e non a eventuali altri tratti compresenti. 


\subsection{Dislocazioni a sinistra e a destra}

I fenomeni di focalizzazione che alterano l'ordine non marcato delle parole costituiscono un tratto distintivo dell'oralità, il "capitolo numero uno" secondo Renzi (2012, p. 41). In particolare, la dislocazione a sinistra, oltre al consueto ruolo di tematizzazione, sta ormai sostituendo la forma passiva, percepita dai parlanti come di registro troppo elevato (ivi, p. 42). La dislocazione a destra è più diffusamente avvertita come struttura ridondante, dunque sanzionata negativamente dal sistema scolastico e, di conseguenza, dai partecipanti al sondaggio. Nel primo assaggio qui proposto, comunque, tutti gli esempi sono tratti del testo originale: non è stata necessaria alcuna aggiunta "in laboratorio". Ciò qualifica già in partenza la dislocazione come struttura ben ambientata nell'italiano giornalistico; fa eccezione l'esempio 5 , ma è difficile spiegarne le cause: né l'assenza della virgola prima dell'elemento dislocato (comune all'esempio 3), né la brevità di quest'ultimo (come nell'esempio 4) sembrano sufficienti a giustificare la minore accettabilità.

\section{a) Dislocazioni a sinistra}

1) E infatti questi quattro bambini li ha schiacciati sulla balaustra proprio in quel punto.

nessuna censura

2) Perché quei viaggi li ho fatti per partecipare a convegni in cui si parla di processi di radicalizzazione, di foreign fighters, di terroristi, delle ragioni di seduzione del Califfato.

nessuna censura

b) Dislocazioni a destra

3) Marianna se la ricorderà per sempre la sua prima vacanza da "grande", con le amiche e senza i genitori 
4) Ma anche una diversa consapevolezza dello spessore della vita, se sai che potresti non ritornarci, a casa: e una gratitudine nuova, quando rivedi i tuoi cari.

nessuna censura

5) Rashid spiega di ricordarle ancora le grida di Mohamed.

6 censure

\subsection{Focalizzazioni}

Sabatini (1985, p. 161) nota come le grammatiche tradizionali abbiano sempre condannato come pleonastiche le costruzioni con ripresa del tema e come francesismi quelle con inizio "è a lui che" ecc. Come rileva poi Berruto (1987, p. 65), tali fenomeni sono in realtà in via di standardizzazione, se non già compiutamente ambientati nell'uso. Possiamo presentare alcuni dati per quanto riguarda il giudizio dei parlanti su frase scissa, pseudoscissa e struttura del c'è presentativo.

\section{a) Frasi scisse}

6) È Okazaki che sblocca il risultato al 5' girando di sinistro sotto l'incrocio un cross di Fuchs.

nessuna censura

7) È più o meno a quell'epoca che lo intraprese un viaggiatore d'eccezione, il poeta Mikhail Jurevic Lermontov.

nessuna censura

8) Ed è in fondo a una gola nera piena di nebbia, nel punto in cui finisce la Georgia e comincia la Russia, che un ragazzo georgiano aspetta l'attacco dell'Armata Rossa di Mikhail Gorbaciov.

nessuna censura

9) È su questi elementi che la Avanzato, bolognese, costruisce il nuovo romanzo.

1 censura

10) Sono questi ragazzi qui, fisici, ingegneri, geologi, a vigilare sull'Italia. nessuna censura 
11) Ogni anno partono 60-90 avvisi di allerta meteo, ed è da questo ufficio che domenica, alle 14, ne è partito uno diretto alla Sardegna.

nessuna censura

12) È lei che firma gli avvisi, che dunque si prende la responsabilità di un atto che può salvare delle vite ma anche generare polemiche.

1 censura

13) È Ronaldo che dà il segno alla gara.

nessuna censura

14) Ora è l'Inter che riesce a spingere indietro l'avversario, che fa anche vedere di saper fare bene il lavoro in difesa.

nessuna censura

b) Pseudoscisse

15) Il loro affetto è stata la cosa che più mi ha emozionato.

nessuna censura

16) Chi sblocca la partita è Cambiasso...

nessuna censura

17) Come già all'inizio del campionato Cambiasso paga il gol e deve lasciare il campo zoppicando. [...] Chi prende il suo posto è Dacourt, e l'Inter non ci rimette.

nessuna censura

18) Chi solleva il caso sono Api-Napoli, Confapi-Campania e i costruttori Aniem, le tre associazioni di categoria autrici della denuncia.

nessuna censura

19) Chi sembra un pesce fuor d'acqua è invece Muntari. nessuna censura

20) Tutto quello che mi interessa è solo lavorare per il bene della città. nessuna censura 
c) $C$ 'è presentativo

21) C'è una finestra, vicino a una delle tante curve che il Tevere fa prima di entrare nel cuore di Roma, che si apre su tutti i cieli d'Italia.

1 censura

22) E ci sono 20 persone che hanno il compito di avvertire il Paese un giorno prima.

1 censura

23) Ma c'è anche qualcosa che la Nazionale ha guadagnato, certamente non tutto qui, ma che qui appieno si è rivelato.

1 censura

24) $\mathrm{Al}$ contrario, c'è qualcuno che ritiene poco incisiva la linea Armato.

nessuna censura

25) C'è Marty che segna ancora.

nessuna censura

26) C'è una 127 bianca con una persona al volante che è ferma nella corsia degli autobus.

nessuna censura

Tutto sommato, tematizzazioni e topicalizzazioni sono accettate quasi universalmente dagli informanti. Anche quando gli articoli proposti alla valutazione sono accusati dai partecipanti al sondaggio di essere scritti in un italiano di registro troppo basso, trascurato, le segnalazioni di scarsa o nulla accettabilità di queste costruzioni sono sporadiche.

\subsection{Oscillazione tra congiuntivo e indicativo}

I cosiddetti "grammarnazi” (Gheno, 2016) e i censori della rete sono particolarmente sensibili al fenomeno chiamato "scomparsa del congiuntivo", "crisi del congiuntivo" ecc.: questo è anche uno degli argomenti principe delle discussioni più catastrofistiche sullo stato della lingua italiana e sul suo futuro. Presupposto dell'indagine è che gli informanti possano perciò dimostrarsi particolarmente sensibili alla sostituzione del congiuntivo con l'indicativo. 
L'indicativo non intacca il congiuntivo quando questo è in una proposizione reggente; nelle subordinate, invece, può prevalere un'interpretazione semantica (indicativo come "modo della certezza" e congiuntivo come "modo dell'incertezza"; Schneider, 1999) oppure sintattica (congiuntivo come espressione di dipendenza, oppure usato per innalzare il registro; Sgroi, 2013). In dettaglio:

27) Sembrava di essere alla corsa dei tori di Pamplona, non capivamo cosa stava succedendo, abbiamo ipotizzato un incendio.

4 censure

28) Chi passava temeva per la propria incolumità visto che non capiva esattamente cosa stava succedendo.

8 censure

29) Voleva sapere dov'era stata e con chi e perché.

1 censura

30) Le indagini proseguono per identificare i due complici che indossavano maschere e parrucche e per verificare se nel centro massaggi sono avvenuti altri episodi analoghi.

1 censura

31) Philippe Villiers è un chirurgo ed è un padre che ieri ha raggiunto l'ospedale senza sapere se i suoi due gemelli e la sua compagna erano in salvo. 4 censure

32) Gli viene chiesto cosa ha visto durante questa notte infinita trascorsa in sala operatoria.

3 censure

33) Io e i miei uomini eravamo certi che si nascondeva lì ma avevamo anche paura di avere fallito come tante altre volte.

2 censure

34) Non c'è stato neanche bisogno di chiedergli se era Bernardo Provenzano. 1 censura

35) Dopo anni ed anni di studio del suo profilo e della sua psicologia, sapevo chi era quell'uomo ormai anziano.

2 censure 
36) Potergli offrire strumenti che li sostengono nella crescita umana e professionale va a beneficio di tutti.

nessuna censura

37) Fonti di polizia e intelligence sostengono che dalla casa di Route de Turin, insieme a un computer portatile e a uno scatolone di carte, sono usciti indizi che fanno dire al primo ministro Manuel Valls che la storia di Mohamed non sia solo affare psichiatrico.

nessuna censura

Come si può vedere, l'indicativo risulta fortemente censurato dopo strutture negative $(27,28,31)$. Il caso 37 (mancato congiuntivo dopo verbi di opinione) rimanda alla notazione di Lepschy \& Lepschy (1988, p. 204) e Renzi (2012, pp. 52-53), in base a cui la scelta congiuntivoindicativo è di tipo stilistico.

\subsection{Che polivalente}

La polivalenza della congiunzione che è stata articolata da Sabatini (1985, p. 164) in almeno quattro tipi principali: temporale, connettore di frase scissa, connettore generico, causale/finale/consecutivo. Le grammatiche, riferisce Sabatini (1985, p. 180), definiscono "abnorme" questo tratto; ma l'autore non ha verificato disparità di giudizio relativamente ai quattro tratti. Secondo D'Achille (1990, p. 212) il che temporale è ammesso dalla norma; al contrario, sono condannati i suoi usi come relativo obliquo. In particolare, la forma radicale (la valigia che ho messo $i$ vestiti) non è stata neppure proposta al giudizio dei nostri informanti, mentre è più diffusa nella varietà media la forma con ripresa pronominale (la valigia che ci ho messo $i$ vestiti), segnalata da Simone (1993, p. 93). Di seguito gli esempi sottoposti alla valutazione; al termine della frase è indicata, per comodità di ragionamento, la funzione del che.

38) Qui la strada è stretta e accidentata tra le pendici delle montagne che un solo uomo basta a fermare un esercito. (consecutivo)

3 censure

39) I russi vengano pure, che noi siamo pronti a morire. (consecutivo)

1 censura 
40) Cosicché lei, biondissima, bellissima, corteggiatissima, si ritrova a dover dipendere in tutto da una zia che Gloria addirittura ne ignorava l'esistenza. (relativo)

6 censure

41) Forse il giorno che sono diventati dodici uguali. Forse il giorno che è arrivato Vullo. Forse il giorno che hanno vinto tanto senza incontrare ostacoli. (temporale)

3 censure

42) In tutte e 4 le stazioni che hanno vinto gli appalti hanno fatto delle cose sporche, sporche, sporche. (relativo dal contesto in cui era inserita la frase emergeva il valore obliquo - in cui).

2 censure

43) L'intercessione del parroco della chiesa dell'istituto che vi andava a scuola la figlia più grande ha convinto le famiglie a tenere un'unica funzione. (spaziale)

8 censure

44) Sotto la lente degli investigatori anche il giorno che Agrosì ha deciso di uccidere la famiglia e poi togliersi la vita... (temporale)

4 censure

45) Avviene, in sostanza, - spiegano Api, Confapi e Aniem - che lo Stato da debitore si trasforma in creditore e chiede il fallimento dell'impresa che lo stesso Stato non le ha ancora pagato i lavori. (relativo)

5 censure

46) Speriamo che i nostri abbiano imparato qualcosa, che sabato c'è un'altra interrogazione. (consecutivo-esplicativo)

2 censure

47) Lui non ascolta, anzi si rimbocca le maniche, che durante le feste il comandante ha sempre molto da fare. (consecutivo-esplicativo)

2 censure e 2 trasformazioni in causale (> ché)

L'uso del che polivalente relativo è ancora oggetto di censura (seppur non maggioritaria, con l'eccezione degli esempi 40 e 43) da parte degli informanti, di ogni età e provenienza geografica. L'uso del che polivalente in funzione consecutivo-esplicativa è sempre segnalato, ma 
da un numero limitato di informanti, che precisano i motivi della correzione. Anche il che temporale non flesso, benché considerato accettabile nello standard, è stato oggetto di correzione, soprattutto da parte di informanti di età adulta.

\section{5. $C i$ attualizzante}

Come già segnalava Sabatini (1985, p. 180), il ci ha talora perduto il suo valore locativo per assumere funzioni di rinforzo semantico e fonico dei verbi essere e avere, e anche di altri verbi. Trent'anni dopo, tuttavia, soltanto alcune grammatiche descrivono il fenomeno, e spesso in maniera rapida e poco dettagliata. Berruto (1987, p. 76) indica una possibile articolazione del fenomeno in casi parzialmente diversi (e tuttavia in parte sovrapponibili). I verbi pronominali formati con il ci possono: (a) possedere una specializzazione semantica rispetto al corrispondente non pronominale; (b) essere sostanzialmente sinonimici, ma con una sfumatura rafforzativa; (c) manifestare un pleonasmo (se il complemento è espresso: ma tu ci credi all'oroscopo?), e in questo caso le frasi possono essere catalogate come dislocazioni, come avviene con il ne. Berruto distingue comunque due possibili valutazioni: per lo standard (c) è costruzione marcata, mentre nel neostandard il pronome fa parte del verbo e il suo uso è una segnalazione di caso (quando la lessicalizzazione del verbo è in fase avanzata). Dopo ogni esempio è indicata tra parentesi la tipologia.

48) Il Bruges però non ci sta e reagisce. (a)

nessuna censura

49) Una volta era solo un sentiero di montagna, e ci voleva un mese di marce forzate per completare il percorso. (a)

nessuna censura

50) Sì, perché dopo il brutto incidente stradale, Gloria si risveglia e non ci vede più. (b)

nessuna censura

51) In una parola deve fidarsi. Che non è mai semplice, ma qui diventa impossibile, specie dopo che ci scappa pure il morto. (a) nessuna censura 
52) Lui, nonostante gli errori che ha fatti, alla necessità di una squadra sognante ci crede ancora. (c)

2 censure

53) Io c'ho un problema grosso, il costruttore Btp. (b)

5 censure: per un informante piemontese è "dialettismo"

54) È l'11': l'Inter c'ha la gara in mano. (b)

8 censure

55) Chi prende il suo posto è Dacourt, e l'Inter non ci rimette. (a) nessuna censura

56) Gli spagnoli non ci capiscono molto, scelgono di interrompere il gioco ad ogni occasione. (a)

2 censure

57) Sul riammodernamento del Vecchio di piazza Miraglia non ci conta. (c) 1 censura

58) Oh, mi sa che ci ho un film da fare. (b)

6 censure (2 affermano che l'espressione sarebbe troppo colloquiale per un giornale)

59) Non mi sarei mai perdonato se avessi sbagliato qualcosa: a quella storia ci tenevo troppo. (c)

1 censura per ordine sbagliato delle parole

60) Quando scrivi ci metti sempre qualcosa di te stesso. (a)

3 censure

Il $c i$ viene censurato soprattutto nella sua casistica di elemento rafforzativo (b) o pleonastico (c). Tuttavia rimane incerta la catalogazione esatta del fenomeno a causa della possibile interpretazione sintattica, come dislocazione, di alcune costruzioni. Al fine di chiarire la questione, sarebbero auspicabili ulteriori indagini volte a indagare l'accettabilità del $\mathrm{ci}$ apparentemente pleonastico in unione a verbi con i quali non è in corso un evidente processo di lessicalizzazione, nonché l'accettabilità di costruzioni dislocate con altri tipi di clitico di ripresa. 
Il $c i$ intensificativo ha uno status più discusso: con vederci o capirci rileviamo poche sanzioni, o nessuna, mentre con il verbo avere la situazione è molto diversa. Anche la diversa scrittura ( $c^{\prime} h o$ oppure il più rispettoso dell'ortografia italiana $\mathrm{ci} h \mathrm{~h}$ ) non modifica l'atteggiamento degli informanti: si dovrà ampliare l'indagine anche in questa direzione, aggiungendo forme come ciò/ciai/cià.

\section{CONCLUSIONI}

Evitiamo in questa sede di fornire dati numerici per il semplice motivo che la modalità stessa con cui si è svolto il sondaggio non permette una quantificazione agevole delle risposte. A differenza dell'approccio adottato da Palermo (2010), infatti, l'articolazione interna delle osservazioni degli informanti è difficilmente riconducibile a misurazioni univoche. Per fare un esempio, tra quelle che al $\S 3$ sono state riportate come "censure" è possibile distinguere le segnalazioni di completa inaccettabilità del costrutto (fino all'agrammaticalità) e commenti che ne segnalano la marcatezza (più o meno spiccata) in diafasia, diamesia e, talvolta, anche in diatopia. Peraltro, come già segnalato al $\S 2$, è anche capitato che i medesimi tratti risultassero meno frequentemente censurati se erano inseriti in citazioni virgolettate, che quindi facevano pensare alla mimesi dell'oralità, o all'interno di testi normalmente caratterizzati da un registro più basso (cronache sportive, pezzi di costume).

Tuttavia è possibile tirare le somme di questo studio preliminare e raggruppare, pur con innegabile approssimazione, i tratti dell'IUM considerati nel nostro sondaggio in tre macrocategorie: quelli che non vanno incontro ad alcuna censura o sono oggetto di segnalazioni minoritarie; quelli che invece vengono segnalati con costanza e, infine, quelli che registrano una certa variabilità delle risposte sulla base del co-testo in cui sono inseriti o delle caratteristiche diastratiche dei rispondenti.

In generale, del primo gruppo fa parte la maggioranza dei fenomeni di tematizzazione e topicalizzazione. Sembra infatti che, almeno nell'italiano dei giornali, la segmentazione (frase scissa e pseudoscissa, c'è presentativo) e la dislocazione a sinistra (meno quella a destra) non vengano più nemmeno percepite come marche diamesiche o di registro. 
Probabilmente si tratta di costrutti che, non essendo sanzionati in contesto scolastico, non influiscono sui giudizi di accettabilità anche quando il parlante viene stimolato a "criticare e correggere" un testo scritto di media formalità: semplicemente, non ci si accorge nemmeno della loro presenza. Discorso analogo vale per le concordanze ad sensum e per la sostituzione del pronome dimostrativo neutro ciò con questo o quello; inoltre quello sembra aver ormai ampliato il proprio campo d'impiego a scapito degli articoli determinativi, soprattutto quando introduce un sostantivo determinato da una relativa (non smetterà mai di chiedere la verità per tutte quelle persone che, come lui, non hanno avuto ancora giustizia) o da espressioni con valore restrittivo (dove sono finiti quei soldi avuti da istituti finanziari e dall'Inps), e non viene di norma sanzionato. Emerge invece una certa insofferenza per il rafforzamento del dimostrativo.

Vengono inoltre segnalati come errati il che polivalente, non solo nei suoi usi più marcatamente sub-standard (come pronome relativo non flesso nei casi obliqui) ma anche quando viene impiegato con valore temporale, sebbene la maggioranza dei soggetti che lo sostituiscono con in cui si raggruppi nella fascia di età compresa tra i 50 e i 65 anni. Proseguendo con l'elenco dei costrutti che la maggioranza degli intervistati avverte come scorretti, menzioniamo l'accordo del participio passato col pronome relativo oggetto diretto (un costrutto indicato come standard da Sabatini, 1985).

Anche il pronome dativale maschile gli in sostituzione del femminile $l e$ incontra la generalizzata riprovazione dei partecipanti al sondaggio, mentre gli al posto del plurale loro risulta più accettabile soprattutto per i giovani tra i 18 e i 25 anni, quando invece gli adulti che lo segnalano nei loro commenti sono due volte più numerosi (10 contro 5$)$. Un altro tratto la cui valutazione richiede di tenere conto del contesto sintattico (in interrogative indirette, dopo verbi che esprimono incertezza, con verbi di opinione e nelle relative restrittive) è l'alternanza indicativo/congiuntivo, che per l'appunto registra giudizi molto variabili e si configura come fatto di registro. Infine, anche l'accettabilità del $c i$ attualizzante dipende dal co-testo: se è possibile interpretare la presenza del clitico come ridondanza pronominale, il $c i$ viene raramente segnalato (coeren- 
temente con i giudizi sulle dislocazioni), mentre negli altri casi le risposte dipendono molto dal verbo a cui si accompagna, probabilmente spia di un processo di lessicalizzazione più o meno avanzato. In ogni caso, il $c i$ è stato sempre rigettato in combinazione con il verbo avere.

Per concludere, se da una parte il nostro metodo di sondaggio non permette di giungere a dati numerici esatti, dall'altra la libertà concessa agli informanti ha permesso di individuare altri fenomeni suscettibili di censura in base alla norma interiorizzata. Non è una sorpresa che gran parte delle osservazioni si siano agglutinate attorno a "regole" che tradizionalmente contraddistinguono l'italiano scolastico, come la condanna di $e$ e $m a$ a inizio frase (Sabatini, 1997) e l'idiosincrasia per le ripetizioni, con la conseguente ricerca insistita della variatio sinonimica, soprattutto tramite la selezione di alternative lessicali di registro elevato (Cortelazzo, 2000b); a questi si aggiunge la critica nei confronti di due caratteristiche fondanti dello stile giornalistico, cioè l'uso espressivo della punteggiatura (origine di molte frasi che iniziano con e e $m a$; cfr. Gualdo, 2007, p. 81 e Cardinale, 2011, p. 145) e l'alta frequenza di frasi nominali e monoproposizionali (che porta ad allontanarsi dal periodare ampio incoraggiato nei temi scolastici). Meno ricorrenti le osservazioni che riguardano altri tratti derivanti dai processi di ristandardizzazione, come l'impiego del futuro epistemico.

\section{BIBLIOGRAFIA}

Acquaviva, P. (2000). La grammatica italiana: il lavoro comincia adesso. Lingua e stile, 2, 249-272.

Antonelli, G. (2007). L'italiano nella società della comunicazione. Bologna: Il Mulino.

Antonelli, G. (2011). Lingua. In A. Afribo \& E. Zinato (eds.), Modernità italiana. Cultura, lingua e letteratura dagli anni Settanta a oggi (pp. 15-52). Roma: Carocci.

Berruto, G. (1987). Sociolinguistica dell'italiano contemporaneo. Roma: La Nuova Italia Scientifica.

Berruto, G. (2012). Sul parlante nativo (di italiano). In Idem, Saggi di sociolinguistica e linguistica (pp. 87-99). Alessandria: Edizioni dell'Orso. 
Cardinale, U. (2011). Manuale di scrittura giornalistica. Torino: UTET Università.

Castellani, A. (1991). Italiano dell'uso medio o italiano senza aggettivi? Studi linguistici italiani, 17, 233-256.

Cortelazzo, M. (2000a). La lingua italiana di fine millennio. In Idem, Italiano d'oggi (pp. 9-24). Padova: Esedra.

Cortelazzo, M. (2000b). Per la storia dell'italiano scolastico. In Idem, Italiano d'oggi (pp. 91-109). Padova: Esedra.

Cortelazzo, M. (2012). Evoluzione della lingua, percezione del cambiamento, staticità della norma. In C. Di Benedetto, S. Ondelli, A. Pezzin, S. Tonellotto, V. Ujcich \& M. Viale (eds.), I sentieri della lingua. Saggi sugli usi dell'italiano tra passato e presente (pp. 15-20). Padova: Esedra.

D’Achille, P. (1990). Sintassi del parlato e tradizione scritta della lingua italiana. Analisi di testi dalle origini al secolo XVIII. Roma: Bonacci.

D'Achille, P., Proietti, D. \& Viviani, A. (2005). La frase scissa in italiano: aspetti e problemi. In P. D'Achille \& I. Korzen (eds.), Tipologia linguistica e società. Considerazioni inter-e intralinguistiche. Due Giornate italo-danesi di studi linguistici (Roma, 27-28 novembre 2003) (pp. 249-279). Firenze: Cesati.

D'Achille, P. (2010), Lingua d'oggi. In Enciclopedia dell'italiano, vol. I (pp. 793-799). Roma: Istituto dell'Enciclopedia Italiana.

Dardano, M. (1994). Profilo dell'italiano contemporaneo. In L. Serianni \& P. Trifone (eds.), Storia della lingua italiana, vol. 2, Scritto e parlato (pp. 343-432). Torino: Einaudi.

De Mauro, T. (2014). Storia linguistica dell'Italia repubblicana dal 1946 ai nostri giorni. Roma-Bari: Laterza.

Gheno, V. (2016). Guida pratica all'italiano scritto (senza diventare grammarnazi). Firenze: Cesati.

Gualdo, R. (2007) L'italiano dei giornali. Roma: Carocci.

Lepschy A.L. \& Lepschy, G.C. (1988). La lingua italiana: storia, varietà dell'uso, grammatica. Milano: Bompiani.

Mioni, A.M. (1983). Italiano tendenziale: osservazioni su alcuni aspetti della standardizzazione. In P. Benincà (ed.), Scritti linguistici in onore di Giovan Battista Pellegrini, vol. 1 (pp. 495-517). Pisa: Pacini.

Ondelli, S. \& Pontrandolfo, G. (2014). La negazione multipla nei testi giuridici: veramente non si può negare che sia un tratto caratteristico? Rivista internazionale di tecnica della traduzione, 16, 153-176. 
Ondelli, S. \& Viale, M. (2010). L'assetto dell'italiano delle traduzioni in un corpus giornalistico. Aspetti qualitativi e quantitativi. Rivista internazionale di tecnica della traduzione, 12, 1-62.

Palermo, M. (2010). L'italiano giudicato dagli insegnanti. LId'O - Lingua italiana d'oggi, 7, 241-251.

Renzi, L. (2012). Come cambia la lingua. L'italiano in movimento. Bologna: il Mulino.

Sabatini, F. (1985). L'“italiano dell'uso medio": una realtà tra le varietà linguistiche italiane. In G. Holtus \& E. Radtke (eds.), Gesprochenes Italienisch in Geschichte und Gegenwart (pp. 154-184). Tübingen: Narr.

Sabatini, F. (1990). Una lingua ritrovata: l'Italiano parlato. In V. Coletti, R. Coluccia, P. D'Achille, N. De Blasi \& D. Proietti (eds.), L'italiano nel mondo moderno. Saggi scelti dal 1968 al 2009 (pp. 89-108). Napoli: Liguori.

Sabatini, F. (1997). Pause e congiunzioni nel testo. Quel "ma" all'inizio di frase... In I. Bonomi (ed.), Norma e lingua in Italia: alcune riflessioni tra passato e presente (pp. 113-146). Milano: Istituto Lombardo di Scienze e Lettere.

Schneider, S. (1999). Il congiuntivo tra modalità e subordinazione: uno studio sull'italiano parlato. Roma: Carocci.

Serianni, L. (2006). Prima lezione di grammatica. Roma-Bari: Laterza.

Serianni, L. (2007). La norma sommersa. Lingua e stile, 42, 283-298.

Serianni, L. \& Benedetti, G. (2009). Scritti sui banchi. L'italiano a scuola tra alunni e insegnanti. Roma: Carocci.

Sgroi, S.C. (2013). Dove va il congiuntivo? Ovvero il congiuntivo da nove punti di vista. Torino: UTET.

Simone, R. (1993). Stabilità e instabilità nei caratteri originali dell'italiano. In A.A. Sobrero (ed.), Introduzione all'italiano contemporaneo, vol. 1, Struttura e variazioni (pp. 41-100). Roma-Bari: Laterza.

Tavoni, M. (2002). Caratteristiche dell'italiano contemporaneo e insegnamento della scrittura. In F. Bruni \& T. Raso (eds.), Manuale dell'italiano professionale. Teoria e didattica (pp. 139-152). Bologna: Zanichelli.

Riassunto: A oltre 30 anni dalla pubblicazione dei 35 tratti dell'italiano dell'uso medio (IUM), pur in presenza di successive osservazioni e puntualizzazioni, si può dire che questa etichetta linguistica sia ormai condivisa da tutti gli studiosi. Tuttavia la scuola non sembra essere stata particolarmente ricettiva delle variazioni nella distanza tra la norma e l'uso e continua a imporre una varietà di italiano che ormai può essere considerata a sé stante. Purtroppo mancano studi che permettano di valutare la 
discrepanza tra norma interiorizzata e comportamento linguistico negli italofoni: poco sappiamo di quali tratti dell'IUM passano del tutto inosservati, quali sono rilevati ma ritenuti accettabili e quali ancora censurati.

Questo contributo rende conto di alcuni sondaggi su parlanti italiani diversificati per provenienza geografica, età, sesso e livello di istruzione, chiamati a esprimere un giudizio su testi giornalistici in cui era presente o è stata inserita ad arte una selezione di tratti dell'IUM. I sondaggi non possono ovviamente dirsi esaustivi e rappresentano solo le prime fasi di uno studio più ampio. Ciononostante, oltre a testare le modalità di somministrazione dei questionari e di selezione degli informanti stessi, i risultati evidenziano la forte influenza della norma interiorizzata sui giudizi, influenza che non si limita ai tratti dell'IUM considerati ma che investe tutta una serie di altri fenomeni che rientrano nell'italiano scolastico e che contribuiscono a formare il modello di riferimento vigente presso la comunità italofona.

Parole chiave: italiano dell'uso medio, norma interiorizzata, norma linguistica, norma sommersa, varietà della lingua 